\title{
Solid-State Properties of One-Dimensional Metals Based on bis(oxalato)platinate Anions with Divalent Cations
}

Braude, A.; Carneiro, K.; Jacobsen, Claus Schelde; Mortensen, Kell; Turner, D. J.; Underhill, A. E.

Published in:

Physical Review B

Link to article, DOI:

10.1103/PhysRevB.35.7835

Publication date:

1987

Document Version

Publisher's PDF, also known as Version of record

Link back to DTU Orbit

Citation (APA):

Braude, A., Carneiro, K., Jacobsen, C. S., Mortensen, K., Turner, D. J., \& Underhill, A. E. (1987). Solid-State Properties of One-Dimensional Metals Based on bis(oxalato)platinate Anions with Divalent Cations. Physical Review B, 35(15), 7835-7846. https://doi.org/10.1103/PhysRevB.35.7835

\section{General rights}

Copyright and moral rights for the publications made accessible in the public portal are retained by the authors and/or other copyright owners and it is a condition of accessing publications that users recognise and abide by the legal requirements associated with these rights.

- Users may download and print one copy of any publication from the public portal for the purpose of private study or research.

- You may not further distribute the material or use it for any profit-making activity or commercial gain

- You may freely distribute the URL identifying the publication in the public portal 


\title{
Solid-state properties of one-dimensional metals based on bis(oxalato)platinate anions with divalent cations
}

\author{
A. Braude \\ Laboratory of Applied Physics III, Technical University of Denmark, DK-2800 Lyngby, Denmark \\ and Physics Laboratory I, H. C. Ørsted Institute, University of Copenhagen, Universitetsparken 5, \\ DK-2100 Copenhagen $\emptyset$, Denmark \\ K. Carneiro* \\ Physics Laboratory I, H. C. Ørsted Institute, University of Copenhagen, Universitetsparken 5, \\ DK-2100 Copenhagen $\emptyset$, Denmark \\ C. S. Jacobsen and K. Mortensen \\ Physics Laboratory III, Technical University of Denmark, DK-2800 Lyngby, Denmark \\ D. J. Turner and A. E. Underhill \\ Department of Chemistry and Institute of Molecular and Biomolecular Electronics, University College of North Wales, \\ Bangor, Gwynedd LL572UW, Wales, United Kingdom \\ (Received 8 May 1986)

\begin{abstract}
The crystal structures, superstructures, dc conductivity, optical properties, and thermopower of
\end{abstract}

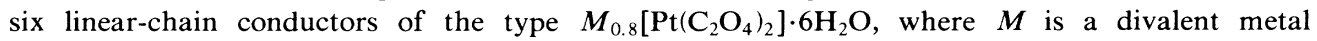 \\ ( $M=\mathrm{Ni}, \mathrm{Co}, \mathrm{Zn}, \mathrm{Fe}, \mathrm{Mg}, \mathrm{Mn}$ ), have been studied. At high temperatures they form a common \\ orthorhombic metallic phase (I) with conductivities of $30-200(\Omega \mathrm{cm})^{-1}$ and thermopowers of 5-10 \\ $\mu \mathrm{V} / \mathrm{K}$, with the lattice weakly modulated by the one-dimensional Peierls distortion. Below $T_{1}$, \\ three compounds $(\mathrm{Ni}, \mathrm{Co}, \mathrm{Zn})$ form a semiconducting phase (A-II) due to the ordering of the \\ $\left[M\left(\mathrm{H}_{2} \mathrm{O}\right)_{6}\right]^{+2}$ cations. As the cation superlattice is commensurate with the three-dimensional \\ Peierls distortion, these salts transform at a lower temperature $T_{2}$ into a charge-density-wave phase \\ (A-III). In the other three compounds ( $\mathrm{Fe}, \mathrm{Mg}, \mathrm{Mn}$ ), the cations order in a superlattice (phase B-II) \\ which is incommensurate with both the average Pt-ion lattice and the three-dimensional Peierls dis- \\ tortion, and therefore no charge-density-wave state is formed. The influence of competing interac- \\ tions is briefly discussed.
}

\section{INTRODUCTION}

Two types of one-dimensional (1D) metals based on nonintegral-oxidation-state platinum complexes have been extensively studied over the past ten years: ${ }^{1,2}$ the aniondeficient tetracyanoplatinates (TCP's) and the cationdeficient bis(oxalato)platinates ( $M$-OP's). The former class is well understood and relationships have been established between the degree of partial oxidation (DPO), the intrachain separation $\left(d_{\|}\right)$and their solid-state properties. $^{1,3}$ In particular, an account has been given of the low-temperature charge-density-wave semiconductor common to all the TCP one-dimensional conductors. This includes an explanation of the regular variation of the activation energy for conduction $\Delta\left(=E_{g} / 2\right)$, as summarized in Fig. 1, which shows the dependence of $\Delta$ upon $d_{\|}$. It also allows a qualitative account of the transition from the 1D metal stable at high temperatures to the 1D semiconductor stable at low temperatures as, for example, represented by the characteristic shape of the conductivity versus inverse temperature. This is illustrated in Fig. 2 for $\mathrm{K}_{2}\left[\mathrm{Pt}(\mathrm{CN})_{4}\right] \mathrm{Br}_{0.3} 3 \cdot 2 \mathrm{H}_{2} \mathrm{O}[\mathrm{KCP}(\mathrm{Br})]$. However, when we tried to apply this detailed knowledge gained
FIG. 1. Activation energy of the low-temperature phase of conducting bis(oxalato)platinates ( $M$-OP), as measured by electrical conductivity, solid circles. For comparison the previously obtained results for the tetracyanoplatinates (TCP) and the fit to a simple charge-density-wave model is shown for comparison. 


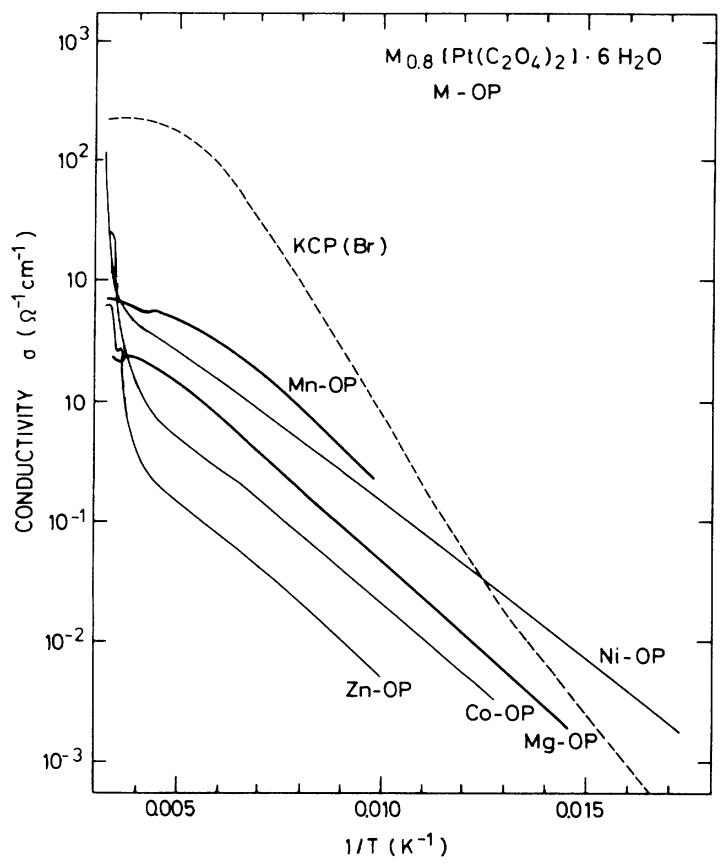

FIG. 2. Temperature dependence of the electrical conductivity for conducting oxalatoplatinates. The result for $\mathrm{KCP}(\mathrm{Br})$ is shown for comparison.

from studies of the $\left[\mathrm{Pt}(\mathrm{CN})_{4}\right]$ conductors to explain the properties for the $\left[\mathrm{Pt}\left(\mathrm{C}_{2} \mathrm{O}_{4}\right)_{2}\right]$ conductors, it became clear that the simple picture has to be modified in several ways. The parameter $\Delta$ (shown in Fig. 1) is significantly larger in the $M$-OP's than expected from the earlier studies of TCP's, although the fact that there is little variation in $\Delta$ is consistent with the similar values of $d_{\|}$in all of the $M$-OP's. A striking difference between the $M$-OP's and the TCP's is the presence of several new features associated with the metal-semiconductor transition in the $M$-OP's which cannot be explained in terms of a simple Peierls instability leading to a charge-density-wave semiconductor.

In this paper we report new results on the structural, infrared reflectivity, conductivity and thermopower properties of several $M$-OP's. These are correlated with previously reported results and an attempt is made to present a coherent picture of the properties of these compounds. We also explain explicitly the difference between this class of compounds and the previously studied TCP's.

\section{PREVIOUS STUDIES OF $M$-OP's}

Although bis(oxalato)platinates of divalent cations ( $M$-OP's) were prepared in the mid 19 th century, it was not until the $\mathrm{x}$-ray structural study of $\mathbf{M g}_{0.82}$ $\left[\mathrm{Pt}\left(\mathrm{C}_{2} \mathrm{O}_{4}\right)_{2}\right] 5 \cdot 3 \mathrm{H}_{2} \mathrm{O}(\mathrm{Mg}-\mathrm{OP})$ by Krogmann that interest in this type of compound was renewed. ${ }^{4}$ Following this work, several $M$-OP's [where $M$ is $\mathrm{Ni}^{5} \mathrm{Co},{ }^{6} \mathrm{Zn},{ }^{7}$ $\mathrm{Fe},{ }^{8} \mathrm{Mn},{ }^{5}$ and $\mathrm{Cu}$ (Ref. 9)] were prepared and characterized. The crystal structure at room temperature has been reported for the Co (Ref. 6) and Ni (Ref. 10) salts and investigations of the superstructure have been carried out on
TABLE I. Crystallographic unit-cell parameters for the orthorhombic (space group $\mathrm{Cccm}$ ) phase I of the conducting salts $M_{0.8}\left[\mathrm{Pt}\left(\mathrm{C}_{2} \mathrm{O}_{4}\right)_{2}\right] \cdot 6 \mathrm{H}_{2} \mathrm{O}(\boldsymbol{M}$-OP). $A$ and $B$ denote the classifications described in the text.

\begin{tabular}{lllllr}
\hline \hline & $a(\AA)$ & $b(\AA)$ & $c(\AA)$ & $V_{c}\left(\AA^{3}\right)$ & Ref. \\
\hline Ni-OP $(A)$ & 16.39 & 14.30 & 5.67 & 1328.5 & 10 \\
Co-OP $(A)$ & 16.501 & 14.379 & 5.682 & 1348.2 & 6 \\
Zn-OP $(A)$ & 16.51 & 14.36 & 5.665 & 1343.9 & 2 \\
Fe-OP $(B)$ & 16.51 & 14.375 & 5.700 & 1352.8 & 8 \\
$\operatorname{Mg}-O P(B)$ & 16.56 & 14.27 & 5.70 & 1347.0 & 4 \\
$\operatorname{Mn-OP}(B)$ & 16.79 & 14.28 & 5.67 & 1359.4 & 2 \\
\hline \hline
\end{tabular}

the compounds of $\mathrm{Ni},{ }^{10,11} \mathrm{Co},{ }^{12-14} \mathrm{Zn},{ }^{15} \mathrm{Fe},{ }^{8}$ and $\mathrm{Mg}$ (Ref. 16) compounds. The variation of electrical conductivity with temperature has been studied for the $\mathrm{Ni},{ }^{10} \mathrm{Co},{ }^{17}, 18$ $\mathrm{Zn},{ }^{7} \mathrm{Mg},{ }^{17,19}$ and $\mathrm{Mn}$ (Ref. 5) salts. In addition, thermopower measurements have been made on the Co (Ref. 5) and $\mathrm{Mg}$ (Ref. 5) salts and optical-reflectivity measurements reported for $\mathrm{Zn}$ (Ref. 7) salt.

We will show that the $M$-OP's can be divided conveniently into two classes on the basis of their structural and transport properties. The class- $A$ compounds are exemplified by Co-OP and the class- $B$ compounds by $\mathrm{Mg}$ OP. However, it is clear that all the $M$-OP's have several properties in common. Only $\mathrm{Cu}-\mathrm{OP}$ which forms a complex structure is an exception, and we will omit a discussion of this compound here. Hence, both Co-OP and $\mathrm{Mg}-\mathrm{OP}$ crystallize in the same orthohombic structure of space group $C c c m\left(D_{2 h}^{20}\right)$ at room temperature. There is little difference in unit-cell parameters, given in Table I,

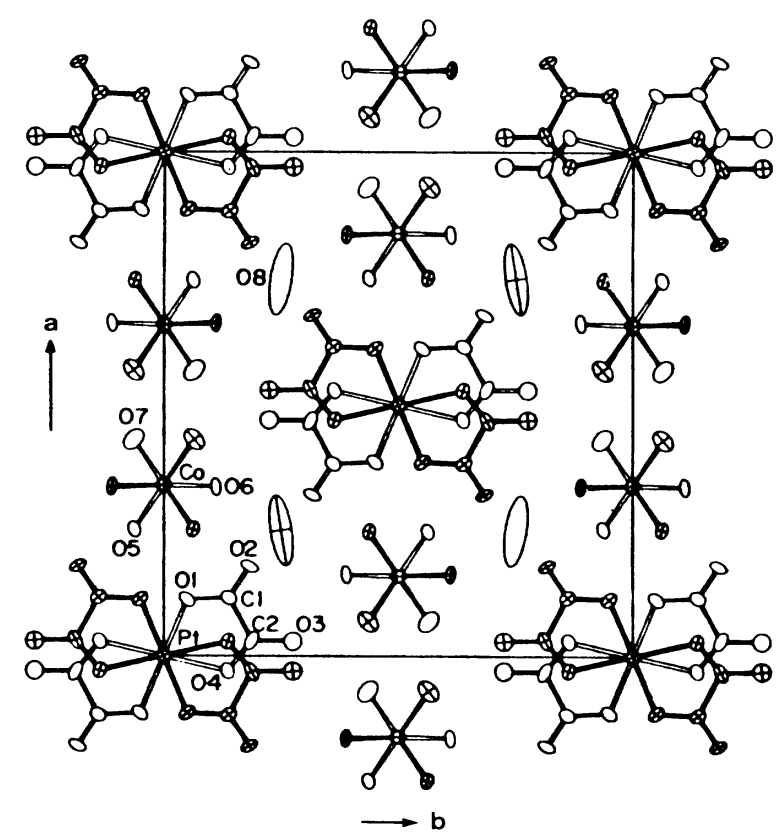

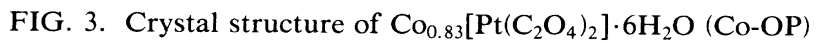
viewed along c. $\left[\mathrm{Pt}\left(\mathrm{C}_{2} \mathrm{O}_{4}\right)_{2}\right]^{-2.34}$ is positioned at $z=0, \frac{1}{2}$; $\left[\mathrm{Co}\left(\mathrm{H}_{2} \mathrm{O}\right)_{6}\right]^{+2}$ is centered at $z=\frac{1}{4}, \frac{3}{4}$, and the extra $\mathrm{H}_{2} \mathrm{O}$ sites (shown by 08) are centered at $z=0, \frac{1}{2}$. Note that $\mathbf{a}$ and $\mathbf{b}$ are chosen according to Refs. 4, 8, and 10 and opposite to Ref. 5. 
or atomic positions for the six salts, although Ni-OP and Mn-OP must be heated above room temperature to form this structure. The common structure is shown for Co$\mathrm{OP}$ in Fig. 3. Both classes exhibit metallic transport properties above room temperature with the characteristics of an almost free-electron-like metal. At some lower temperatures, they both undergo a structural change; but the effect on transport properties is different in the two classes. This is apparent from Fig. 2, where the conductivity of Co-OP drops an order of magnitude around room temperature $\left(T^{-1} \cong 0.003 \mathrm{~K}^{-1}\right)$ as opposed to $\mathrm{Mg}$ OP's which show only a slight increase. At even lower temperatures, they both behave as semiconductors with similar activation energies in the range $50-55 \mathrm{meV}$.

\section{X-RAY STRUCTURE STUDIES}

Earlier studies of Co-OP and $\mathrm{Mg}$-OP indicated that conducting oxalatoplatinates belong to the two classes mentioned above. As we shall see, the different behavior manifests itself in several properties, but the classes are most clearly distinguished from structural investigations by their different lattice modulations. Class $A$, to which $\mathrm{Ni}-\mathrm{OP}$, Co-OP, and Zn-OP belong, exhibits three wellcharacterized phases separated by two transition temperatures, as shown in Fig. 4. Class B, containing Fe-OP, $\mathrm{Mg}-\mathrm{OP}$, and Mn-OP, seem to show only one phase transition, as shown in Fig. 5. However, their low-temperature

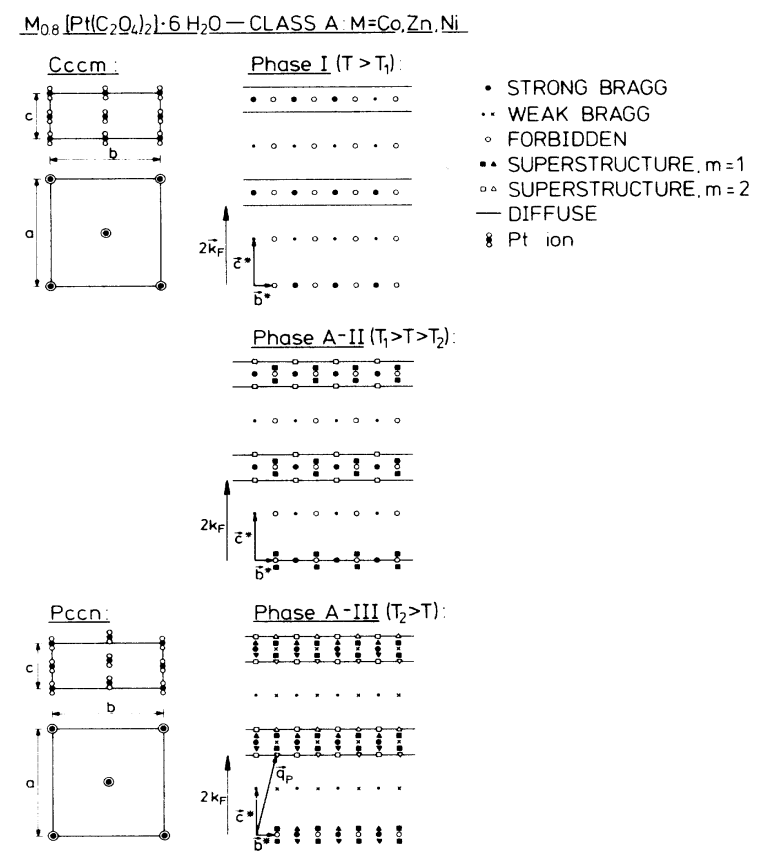

FIG. 4. Structural characteristics of class- $A$ M-OP's. Note that for the low-temperature Pccn structure we have chosen a nonconventional unit cell to make the comparison to the hightemperature $\mathrm{Cccm}$ structure more direct. Satellite reflections are only shown around the strong Bragg-reflection layer lines $(L=0,2,4 ; \mathbf{H} \neq 0)$ and their positions are summarized in Table III.

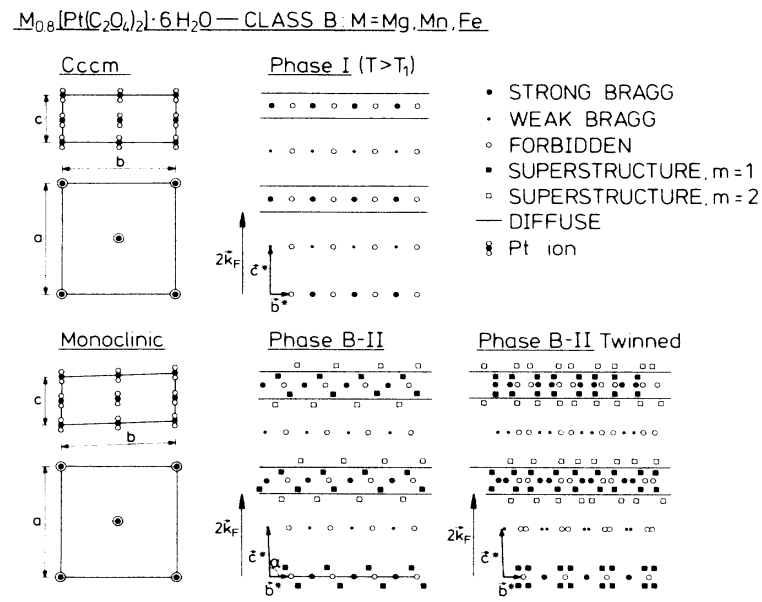

FIG. 5. Structural characteristics of class- $B M$-OP's. Superstructure reflections are only shown around the strong Braggreflection layer lines $(L \sim 0,2,4 ; \mathrm{H} \neq 0)$.

phase cannot be unambiguously characterized, but it is distinctly different from both the low-temperature phases of class $A$.

\section{A. The common high-temperature phase. Phase I}

All the compounds studied here crystallize in the same high-temperature structure, shown in Fig. 3. It belongs to the orthorhombic space group $\mathrm{Cccm}$. Each unit cell includes molecules from two conducting chains. The conducting direction is parallel to $\mathrm{c}$ with two nonequivalent but equidistant $\left[\mathrm{Pt}\left(\mathrm{C}_{2} \mathrm{O}_{4}\right)_{2}\right]$ ions along c. The intrachain Pt separation varies within $d_{\|}=2.84 \pm 0.02 \AA$ for all six compounds studied here (Table I).

In phase $\mathrm{I}$, the $\left[\mathrm{M}\left(\mathrm{H}_{2} \mathrm{O}\right)_{6}\right]^{+2}$ cations are situated in channels parallel to the conducting chains. There are two such channels per Pt chain, giving a random-site occupancy of $0.41-0.42$. However, neighboring channels are so close that it is unlikely that both cation positions are occupied at the same level in the unit cell.

In the structural investigation of Co-OP, an independent water site was found, containing the water molecules which are not coordinated to the $\mathrm{Co}^{+2}$ ion. ${ }^{5}$ This, together with thermogravitometric analysis, indicated that the chemical formula is $\mathrm{Co}_{0.83}\left[\mathrm{Pt}\left(\mathrm{C}_{2} \mathrm{O}_{4}\right)_{2}\right] \cdot 6 \mathrm{H}_{2} \mathrm{O}$. It was concluded that this extra water position was probably overlooked when the structure of $\mathrm{Mg}$-OP was solved. ${ }^{4}$ As we shall see, the possibility of accumulating "extra" $\mathrm{H}_{2} \mathrm{O}$ or $\mathrm{H}_{3} \mathrm{O}^{+}$in the crystal may play a crucial role in the distinction between the class- $A$ and - $B$ compounds.

At high temperatures, the linear Pt chains are modulated only by a one-dimensional sinusoidal distortion due to the Peierls instability. Although phase $I$ is metallic in its transport properties, precursor effects of this instability show up as diffuse $x$-ray scattering at scattering angles where the $c$ component of the wave-vector transfer is $2 k_{F}$, where $\mathbf{k}_{F}$ is the electronic Fermi vector.

Figures 4 and 5 show the $C c c m$ unit cell and the $\left(b^{*}, c^{*}\right)$ reciprocal-lattice plane of the common phase I. 
TABLE II. Transition temperatures $T_{1}$ and $T_{2} . \quad T_{1}$ separates phase I (Peierls distorted metal) and phase II (which has molecular ordering). $T_{2}$ marks the Peierls transition in class- $A$ compounds

\begin{tabular}{lccc}
\hline \hline Compound & Class & $T_{1}(\mathrm{~K})$ & $T_{2}(\mathrm{~K})$ \\
\hline Ni-OP & $A$ & 315 & 301 \\
Co-OP & $A$ & 303 & 280 \\
Zn-OP & $A$ & 303 & 278 \\
Fe-OP & $B$ & 273 & \\
Mg-OP & $B$ & 284 & \\
Mn-OP & $B$ & $>300$ & \\
\hline
\end{tabular}

The transition temperature $T_{1}$, below which phase I becomes unstable, is shown in Table II. Note again that Ni-OP and Mn-OP only exhibit phase I above room temperature.

\section{B. Class- $\boldsymbol{A}$ compounds: Phase A-II}

Below the temperature $T_{1}$, the structures of Ni-OP, $\mathrm{Co}-\mathrm{OP}$, and $\mathrm{Zn}-\mathrm{OP}$ become modulated, corresponding to the formation of an incommensurate superlattice, without any change in space group of the underlying average lattice. As a result of this transition, satellite reflections occur at wave vectors $\pm \mathbf{k}_{F}$ around forbidden Bragg reflections and at $\pm 2 \mathbf{k}_{F}$ around strong Bragg reflections of the $\mathrm{Cccm}$ space group. The $\mathrm{x}$-ray diffraction pattern from $\mathrm{Zn}-\mathrm{OP}$ is shown in Fig. 6. The two types of satellite reflections may be conceived as originating from wave vectors in $m\left(\mathbf{b}^{*}+\mathbf{k}_{F}\right)$, with $m= \pm 1, \pm 2$. First-order reflections $(m= \pm 1)$ are shown in Fig. 4 as solid squares, $\boldsymbol{\square}$, and second-order ( $m= \pm 2$ ) by open squares, $\square$, and their positions are summarized in Table III. Similarly, one identifies another wave vector $m\left(\mathbf{a}^{*}+\mathbf{k}_{F}\right)$ stemming from a separate set of satellite reflections. During the transition, there is no change in intensity of the $2 k_{F}$ diffuse scattering. The intensity distribution of the satellite reflections indicates that the amplitudes of the distortion are polarized along the $\mathbf{b}$ axis.

Obviously, it is a very difficult task to describe the details of the incommensurate superstructure observed in phase A-II, and several somewhat different explanations have emerged. With respect to the one-dimensional Peierls instability, it should be noted that the transition at $T_{1}$ is three-dimensional in nature and is characterized by wave vectors that do not destroy the Fermi surface by a simple Peierls transition. Phase A-II must therefore be characterized by some kind of molecular ordering, associated with the cations and/or the oxalato ligands, and to make the distinction from the Peierls transition clear, Braude et al. named the transition at $T_{1}$ the "nonPeierls" transition. ${ }^{12}$

In their $\mathrm{x}$-ray studies of $\mathrm{Ni}-\mathrm{OP}$, Kobayashi et al. ${ }^{10}$ described the phase A-II in terms of ordering of the $\left[\mathrm{Ni}\left(\mathrm{H}_{2} \mathrm{O}\right)_{6}\right]^{+2}$ cations and the presence of ordered vacancies in the columns described above. The average distance between vacancies in each channel corresponds to a wave vector $\mathbf{k}_{F}$ along $\mathbf{c}$. Neighboring cation channels are coupled in antiphase. The periodicity of the cation vacancies
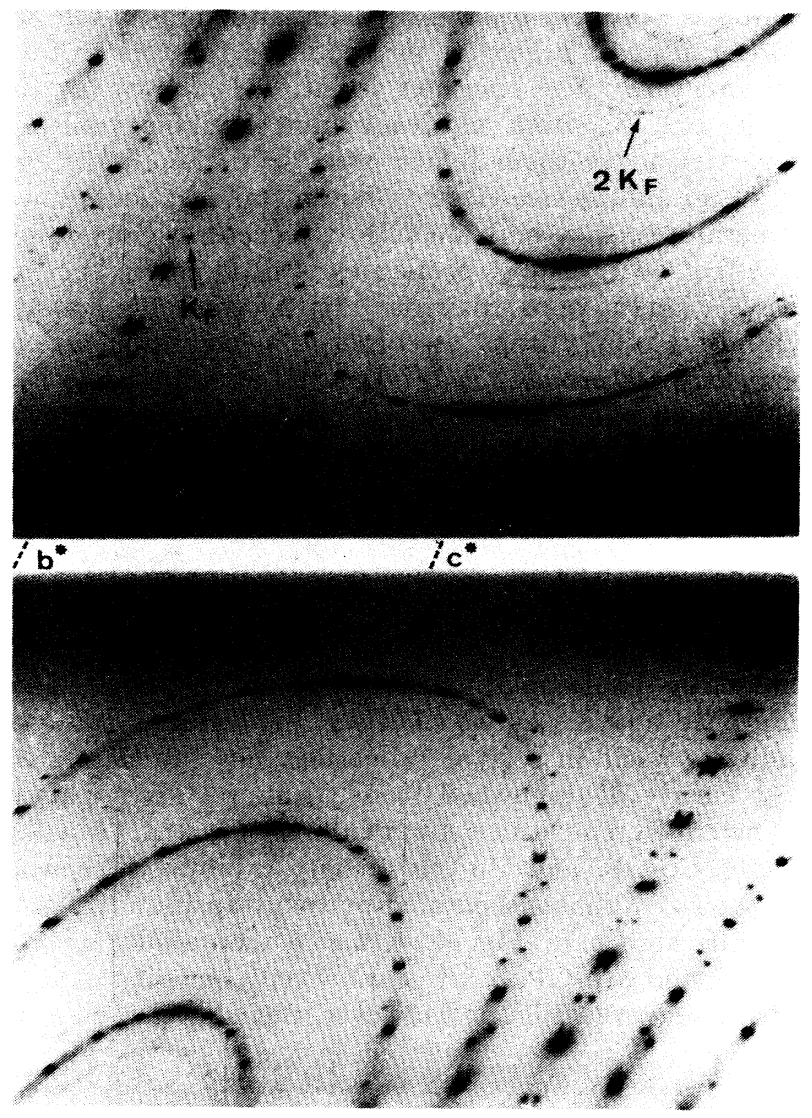

FIG. 6. X-ray Weissenberg recording of $\mathrm{Zn}-\mathrm{OP}$ at $283 \mathrm{~K}$. It shows the $(0 \mathrm{KL})$ plane, containing $2 \mathbf{k}_{F}$ diffuse scattering and second-order satellites, both surrounding strong Bragg-reflection layer lines, as well as first-order spots surrounding extinct Bragg reflections. The picture is characteristic of phase A-II, as shown in Fig. 4.

introduces a deformation of the Pt chain. This is the origin of the transverse component of the modulation.

Berinotti and Luzet, ${ }^{14}$ in a study of $\mathrm{Co}-\mathrm{OP}$, focused upon the spatial arrangement of the $\left[\mathrm{Pt}\left(\mathrm{C}_{2} \mathrm{O}_{4}\right)_{2}\right]$ anions. They proposed that below $T_{1}$, microdomains are formed which are separated by local lattice deformations, leading to a so-called polaron paracrystal. The complexity of the structure of phase A-II is probably related to the need for coexistence between the incommensurate Wigner lattice of

TABLE III. Space groups and super-lattice wave vectors in the three phases of class- $A M$-OP's ( $M=\mathrm{Ni}, \mathrm{Co}, \mathrm{Zn}$ )

\begin{tabular}{llc}
\hline \hline & $\begin{array}{c}\text { Space } \\
\text { group }\end{array}$ & \multicolumn{1}{c}{$\begin{array}{c}\text { Super-lattice } \\
\text { wave vectors }\end{array}$} \\
\hline Phase I & $C c c m$ & $2 \mathbf{k}_{F}$ \\
Phase A-II & $C c c m$ & $2 \mathbf{k}_{F}$ \\
& & $m\left(\mathbf{a}^{*}+\mathbf{k}_{F}\right),|m|=1,2$ \\
Phase A-III & Pccn & $m\left(\mathbf{b}^{*}+\mathbf{k}_{F}\right),|m|=1,2$ \\
& & $2 \mathbf{k}_{F}$ \\
& & $m\left(\mathbf{a}^{*}+\mathbf{k}_{F}\right),|m|=1,2$ \\
& $m\left(\mathbf{b}^{*}+\mathbf{k}_{F}\right),|m|=1,2$ \\
\hline
\end{tabular}


the cations and the regular crystallographic structure formed by the $\left[\mathrm{Pt}\left(\mathrm{C}_{2} \mathrm{O}_{4}\right)_{2}\right]$ ions, a point we shall return to below.

Finally, it should be noted that the cation order in phase A-II leads to semiconducting behavior of transport properties. However, it should not be confused with the Peierls semiconductor, occurring in phase A-III.

\section{Class $-A$ compounds: Phase A-III}

At the temperature $T_{2}$, Ni-OP, Co-OP, and $\mathrm{Zn-OP}$ undergo a second structural phase change into phase A-III. $T_{2}$ varies from $5 \mathrm{C}$ to $28 \mathrm{C}$, as shown in Table II. Below the transition, the two $\mathrm{Pt}$ chains, which are related by the translation vector $\frac{1}{2}(\mathbf{a}+\mathbf{b})$ in the $C c c m$ space group, shift along $\mathbf{c}$ with respect to each other. This gives rise to the appearance of new Bragg reflections (shown by $\times$ in Fig. 4) at $H+K=2 n+1, L \neq 0$, in accordance with the Pccn $\left(D_{2 h}^{20}\right)$ space group.

The satellite reflections of phase A-III are shown in Fig. 4 and may be characterized as follows. New satellite reflections occur at $\pm \mathbf{k}_{F}$ around strong Bragg reflections ( $\triangle$ in Fig. 4) and at $\pm 2 \mathbf{k}_{F}$ around the new Bragg reflections ( $\Delta$ in Fig. 4). These new satellite reflections may be thought of as originating from the new Bragg reflections via the same wave vectors as observed in phase A-II, and therefore the symmetry of the superstructure does not change during the $T_{2}$ transition. The intensities of both the new Bragg reflections and of the four different satellite reflections show a drastic temperature dependence below their respective transition temperatures. Also, their relative strengths vary with temperature. This indicates a continuous but different temperature development of the distortions involved in the transition from A-II to A-III.

Of paramount importance for the electronic properties of $M$-OP's is the observation that the wave vector of the second-order satellites in phase A-III (marked by open triangles in Fig. 4) corresponds to the wave vector of a simple Peierls distortion when referred to the strong Bragg reflections. Hence, with respect to the Peierls instability, the $T_{2}$ transition corresponds to a Peierls transition where the electronic charge-density waves order in a threedimensional array with the same antiphase relationship between neighboring $\mathrm{Pt}$ chains, as observed in $\mathrm{KCP}(\mathrm{Br})$. Accordingly, the conductivity of class- $A$ compounds in Fig. 2 demonstrates an increase in the activation energy, when cooled through the transition at $T_{2}$.

In phase A-III, weak $2 k_{F}$ diffuse scattering remains, which we attribute to the absence of long-range order.

\section{Class- $B$ compounds: Phase B-II}

At $T_{1}, \mathrm{Fe}-\mathrm{OP}, \mathrm{Mg}-\mathrm{OP}$, and $\mathrm{Mn}-\mathrm{OP}$ undergo a crystallographic phase change from the $\mathrm{Cccm}$ orthohombic phase I to a monoclinic phase, phase B-II. The monoclinic distortion occurs in the $\alpha$-angle which assumes the value of about $89^{\circ}$ in $\mathrm{Mg}-\mathrm{OP}$, associated with an in-phase sliding of the $\mathrm{Pt}$ chains as opposed to the antiphase sliding of the chains in phase A-III. The formation of the B-II phase always gives rise to crystallographic twinning of about $2^{\circ}$, which makes an accurate crystal-structure analysis impractical. However, our evaluation of lowtemperature Weisenberg photographs indicates that the space group of the B-II phase (at least in Mg-OP) is most likely $C_{2 / c}\left(C_{2 h}^{6}\right)$ as proposed by Dubois for Mg-OP. ${ }^{16}$

In phase B-II, superstructures occur which are in some respects similar to those observed in phase A-II. In Fig. 5 we show the satellite reflection pattern of phase B-II. This was first analyzed by Dubois in terms of a transverse modulation of the positions of the $\left[\mathrm{Pt}\left(\mathrm{C}_{2} \mathrm{O}_{4}\right)_{2}\right]$ chains ${ }^{16}$ in $\mathrm{Mg}-\mathrm{OP}$, and in this respect the analysis is similar to that by Kobayashi et al. for Ni-OP. However, in contrast to the phase A-II, the modulation wave vector in phase B-II was reported to be incommensurate both with $\mathbf{k}_{F}$ along $\mathbf{c}$ and also with the crystallographic lattice along $\mathbf{a}$ and $\mathbf{b}$. In the twinned crystal, the composite picture becomes rather complicated, as shown in Fig. 5 and in the $x$-ray photographs of Fig. 7. But the simple observation that the second-order satellites do not fall on the diffuse $2 k_{F}$ lines immediately reveals that a Peierls transition as observed for the class- $A$ compounds cannot occur in the phase B-II. Hence, the difference in the periods of their cation order between the two classes of $M$-OP's appear to determine their ability to undergo the metal-insulator transition resulting from the Peierls instability. In consequence, no second-phase change corresponding to the Peierls transition is observed in Fe-OP, Mg-OP, and $\mathrm{Mn}$ OP.

The lack of commensurability between the cation superlattice and the $2 k_{F}$ period of the charge-density wave requires an explanation, since it would appear that the two periods are connected by charge neutrality. However, if $\mathrm{H}_{3} \mathrm{O}^{+}$is formed together with $\left[\mathrm{Mg}\left(\mathrm{H}_{2} \mathrm{O}\right)_{6}\right]^{+2}$ during crystal preparation, then the cation period along $\mathrm{c}$ will be less than $k_{F}$, as was observed by Dubois and later by us. To explore this feature, we have grown crystals of $\mathrm{Mg}$-OP from solutions of different acidity. Indeed, they do show differences in values of unit cell and $2 k_{F}$ parameters and $2 k_{F}$ may be produced, and that they are associated with dramatic changes in the superstructure reflection pattern. In some cases, we find that the period of the superstructure is commensurate with $2 \mathbf{k}_{F}$ along $\mathbf{c}$; but the periods
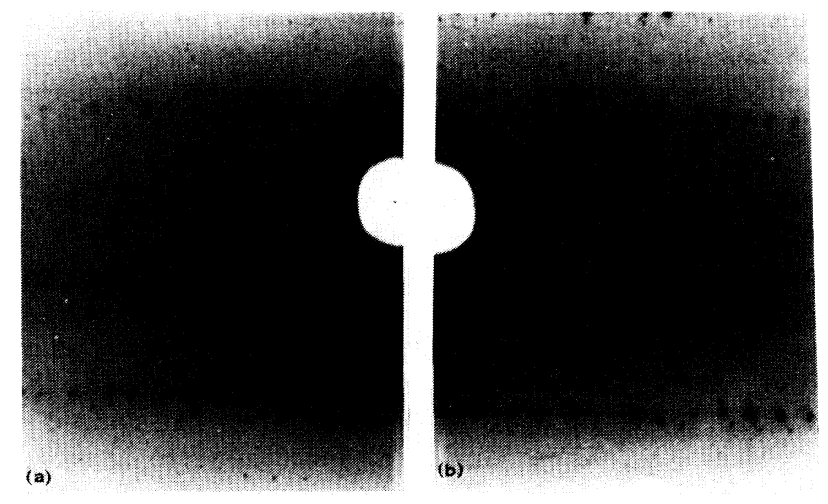

FIG. 7. X-ray photographs showing two different superstructures in one crystal of $\mathrm{Mg}$-OP at $230 \mathrm{~K}$. The two pictures were obtained by focusing the $\mathrm{x}$-ray beam on different parts of the crystal. 
along $\mathbf{a}$ and $\mathbf{b}$ appear far from commensurability. Therefore, the relation to the Peierls transition is the same for all B-II satellite patterns, and no activation energy is expected in the electronic spectrum.

As a further illustration of the delicate interplay between cation order and the Pt lattice, we even observed different superstructures in different parts of one crystal of $\mathrm{Mg}-\mathrm{OP}$, as shown in Fig. 7.

Whereas the class- $A$ compounds Ni-OP, Co-OP, and $\mathrm{Zn}-\mathrm{OP}$ have all been studied by several techniques, only $\mathrm{Mg}-\mathrm{OP}$ of class $B$ has been extensively studied. In MnOP, structural investigations are impeded by deterioration of the crystals by prolonged $\mathrm{x}$-ray exposure, whereas $\mathrm{Fe}$ OP has been well studied by $\mathrm{x}$-rays. ${ }^{8}$

In summary, the phase B-II may be characterized by a monoclinic distortion and a complex set of different superstructures, but none of these correspond to the superstructure of the phase A-II. Since three-dimensional ordering resulting from the Peierls instability requires that both the period along $\mathrm{c}$ be $2 k_{F}$ and that the period perpendicular is $2 \pi / a$ (or $2 \pi / b$ ), none of the different satellite patterns in phase B-II are compatible with such an ordering. Therefore, the Peierls transition is unlikely to occur. This appears to be the likely explanation for the lack of a second transition in class- $B$ components. In consequence, activated behavior is not expected.

\section{OPTICAL REFLECTANCE STUDIES}

In dealing with synthetic metals, polarized reflectance studies constitute the most convenient way of obtaining broad band spectra. In order to illuminate the electronic properties of the conducting bis(oxalato)platinates, and in particular to distinguish between classes $A$ and $B$, we have therefore supplemented the previous reflectance study of Zn-OP (Ref. 7) with measurements on Co-OP, $\mathrm{Mg}-\mathrm{OP}$, and Mn-OP.

\section{A. Results and Drude analysis}

For all materials studied (Co-OP, Zn-OP, Mg-OP, Mn$\mathrm{OP})$, the reflectance component perpendicular to the $\mathbf{P t}$ chains was found to be small $(8-10 \%)$ and rather dispersionless from $5000 \mathrm{~cm}^{-1}$ and throughout the visible. In contrast, the c-axis spectra show a reflectance edge reminiscent of metallic behavior. In Fig. 8, the spectra of Co$\mathrm{OP}, \mathrm{Zn}-\mathrm{OP}, \mathrm{Mg}-\mathrm{OP}$, and $\mathrm{Mn}-\mathrm{OP}$ are shown. The reflectance minimum is at about the same position $\left(19000 \mathrm{~cm}^{-1}\right)$ in all materials, but the sharpness of the edge and of the minimum varies considerably from material to material. Different samples of the same material show fairly reproducable behavior. However, we believe that the smearing of the edge, most drastically observed in $\mathrm{Mn}-\mathrm{OP}$, is due to imperfect surfaces and characteristic for a specific preparation. In this respect, the best sample is that of $\mathrm{Zn}-\mathrm{OP}$, where the reflectance drops to approximately $3 \times 10^{-4}$, one of the deepest plasma minima ever recorded. For $\mathrm{Mg}-\mathrm{OP}$, data are given both at 300 and at $200 \mathrm{~K}$, i.e., both above and below the phase transition observed at $T_{1}=283 \mathrm{~K}$. No significant differences in the optical properties are observed at the two temperatures.

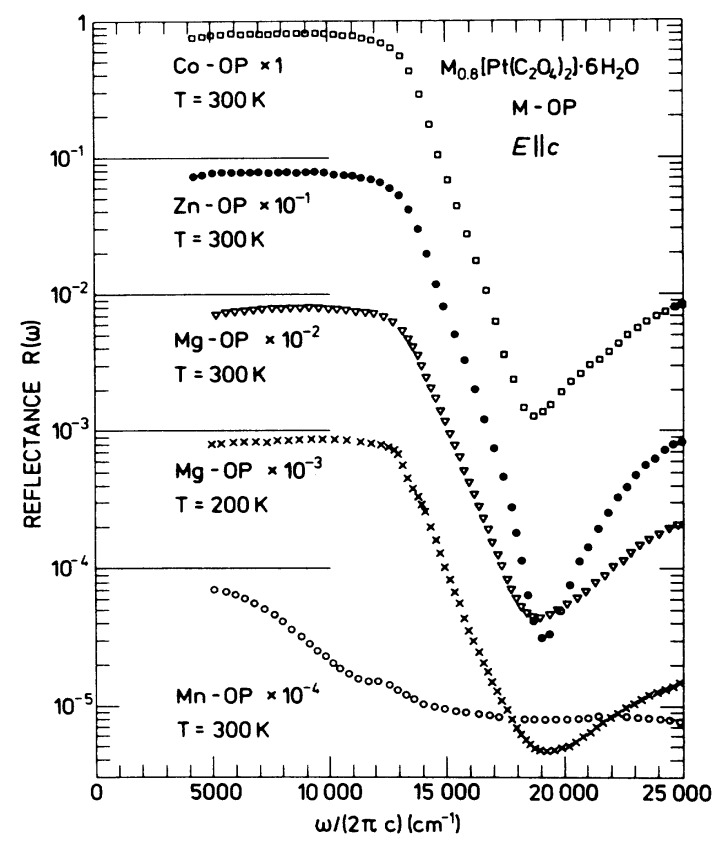

FIG. 8. Polarized reflectance spectra of $M$-OP's in the visible and near-infrared optical region.

In order to extract quantitative information from the data, they have been analyzed according to the Drude theory. ${ }^{20}$ Within this theory, the relative complex dielectric function is given by

$$
\begin{aligned}
\tilde{\epsilon}(\omega) & =\epsilon(\omega) \epsilon_{0} \\
& =\epsilon_{\infty}-\frac{\omega_{p}^{2}}{\omega(\omega+i \gamma)} .
\end{aligned}
$$

Here, $\omega_{p}$ is the plasma frequency, $\gamma$ is a relaxation rate, and $\epsilon_{\infty}$ the background dielectric constant. The frequency for plasma oscillations, the screened plasma frequency, is given by $\Omega_{p}=\omega_{p} / \sqrt{\widetilde{\epsilon}_{\infty}}$. The reflectance is given by:

$$
R(\omega)=\left|\frac{\sqrt{\tilde{\epsilon}(\omega)-1}}{\sqrt{\tilde{\epsilon}(\omega)+1}}\right|^{2} .
$$

The main difficult that arises in fitting (1) and (2) to the data is a high correlation between $\epsilon_{\infty}$ and $\omega_{p}$. Since our main concern is reliable $\omega_{p}$ values, we took the following approach: A good fit was first obtained to the highquality data of $\mathrm{Zn}-\mathrm{OP}$. The value of $\epsilon_{\infty}=2.03$ found for $\mathrm{Zn}$-OP was then applied to the other materials. This is reasonable because of the following:

(1) Solution spectra show that $\left[\mathrm{Pt}\left(\mathrm{C}_{2} \mathrm{O}_{4}\right)_{2}\right]^{2-}$ has absorption bands at much lower energies than $\left[M\left(\mathrm{H}_{2} \mathrm{O}\right)_{6}\right]^{2+}$. Since the low-lying transitions will dominate $\epsilon_{\infty}$, this parameter is presumably rather insensitive to the choice of cation $M$.

(2) Other platinum chain conductors ${ }^{21,22}$ have similar background dielectric constraints, $\epsilon_{\infty}=2.1-2.2$.

Table IV gives the results of the analysis of the data from Fig. 8. We have not attempted to analyze the very 
TABLE IV. Drude parameters for M-OP's $\mathbf{E}|| \mathbf{c}$.

\begin{tabular}{lcccc}
\hline \hline Material & $T(\mathrm{~K})$ & $\frac{\omega_{p}}{2 \pi c}\left(\mathrm{~cm}^{-1}\right)$ & $\frac{\gamma}{2 \pi c}\left(\mathrm{~cm}^{-1}\right)$ & $\epsilon_{\infty}$ \\
\hline Zn-OP & 300 & 19400 & 1200 & 2.03 \\
Co-OP & 300 & 19300 & 2200 & \\
Mg-OP & 300 & 20300 & 1600 & \\
Mn-OP & 200 & 19600 & 700 & \\
\hline \hline
\end{tabular}

broad spectrum of Mn-OP. As noted above, the $\gamma$ values probably have little significance, but simply reflect the sample quality. However, we do see a decrease in $\gamma$ on cooling Mg-OP from 300 to $200 \mathrm{~K}$, as might be expected from decreased electron-phonon scattering. Judging from Fig. 8, the decrease is associated with a sharpening of the reflectance edge at $\omega /(2 \pi c) \simeq 14000 \mathrm{~cm}^{-1}$. The plasma frequency corresponds to $\omega_{p} /(2 \pi c)=19600 \mathrm{~cm}^{-1}$ with only slight variation between compounds. This value is somewhat lower than those found in $\mathrm{KCP}(\mathrm{Br})(23700$ $\mathrm{cm}^{-1}$ ) (Ref. 21) and K(def)TCP $\left(23900 \mathrm{~cm}^{-1}\right) .^{22}$

The screened plasma frequency for the $M$-OP's corresponds to $\Omega_{p} /(2 \pi c) \approx 13800 \mathrm{~cm}^{-1}$. Dispersion analysis of the data in Fig. 8, or in some cases just careful inspection, shows the existence of a weak-absorption band in the $13-14000 \mathrm{~cm}^{-1}$ range $(\simeq 1.7 \mathrm{eV})$. This can hardly be accounted for as an interband transition, and may be assigned to optical excitations of plasmons by the Hopfield mechanism, ${ }^{23}$ as has also been suggested in $\mathrm{KCP}(\mathrm{Br})$ for a band near $18000 \mathrm{~cm}^{-1}$.

The high value of the bare plasma frequency $\omega_{p}$ for the $M$-OP series indicates that their conduction bands have large band widths. Furthermore, the similarity between the spectra in Fig. 8 demonstrates that the infrared properties do not vary significantly between the different phases I, A-II, B-II.

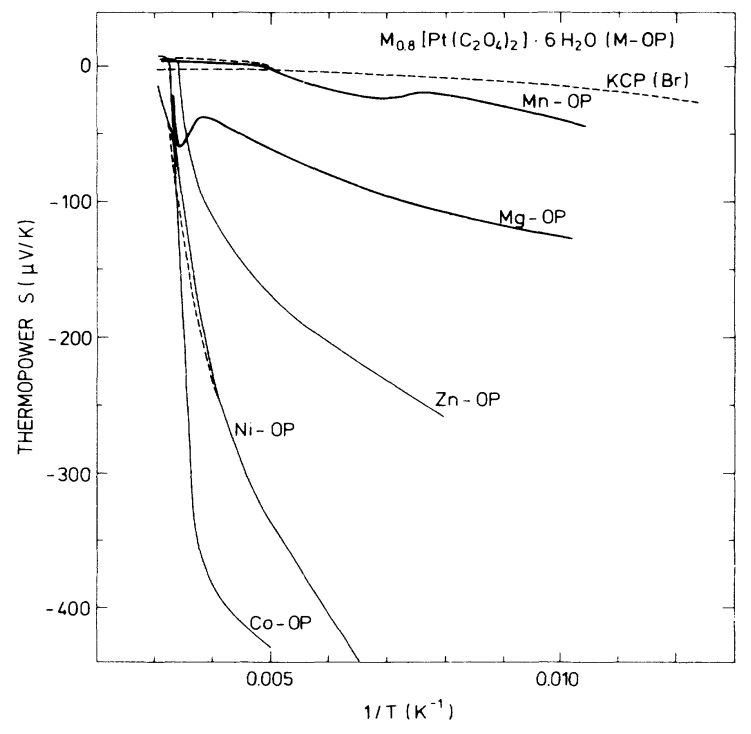

FIG. 9. Temperature dependence of the thermopower of $M$-OP's. The results for $\mathrm{KCP}(\mathrm{Br})$ are shown for comparison.

\section{THERMOPOWER STUDIES}

The thermopower of one-dimensional conductors provides very useful information about band structure, transport properties, and phase transitions. Earlier studies of Co-OP and Mg-OP indicate that the two classes of $M$ OP's have distinctly different thermopowers, ${ }^{5}$ and we have supplemented these results with measurements on Ni-OP, Zn-OP, and Mn-OP. The longitudinal thermopower $S$ was measured in a conventional apparatus, using a slow alternating temperature difference of typically 0.2 $\mathrm{K} .{ }^{24}$ The samples were mounted on $35-\mu \mathrm{m}$ gold wires and contacts were made using Aquadag. In order to prevent dehydration of the crystals, the measurements were made with the sample chamber open to the atmosphere at high temperatures. When the temperature was well below 273 $K$, the thermopower of the samples was unaffected by evacuating the chamber, and below $240 \mathrm{~K}$, the measurements were performed in vacuum. Figure 9 summarizes our results, and below we describe them with reference to the crystallographic phases presented above.

\section{A. Results for phase I}

At high temperatures above $T_{1}$, the thermopowers of Co-OP, $\mathrm{Zn}-\mathrm{OP}, \mathrm{Mg}-\mathrm{OP}$, and $\mathrm{Mn}-\mathrm{OP}$ are small and positive, $S=5-10 \mu \mathrm{V} / \mathrm{K}$, and typically with a linear temperature dependence $d|S| / d T \simeq 40 \mathrm{nV} / \mathrm{K}^{2}$. The apparent deviation from this picture of Ni-OP in Fig. 9 is ascribed to the high $T_{1}$ so that the samples cannot be examined well above $T_{1}$ without deterioration. The same is true for Mn-OP, which easily loses its crystal water.

Based on transport theory in one dimension, the thermopower is given by the following expression: ${ }^{25}$

$$
S=-\frac{\pi^{2}}{3} \frac{k_{B}}{e} k_{B} T\left[\frac{\varepsilon^{\prime \prime}(k)}{\left[\varepsilon^{\prime}(k)\right]^{2}}+\frac{\tau^{\prime}(\varepsilon)}{\tau(\varepsilon)}\right]_{k=k_{F}},
$$

where $\varepsilon^{\prime}(k)$ and $\varepsilon^{\prime \prime}(k)$ are the first and second derivatives with respect to the wave number of the electronic energy dispersion relation characterizing the band structure. $\tau^{\prime}(\varepsilon)$ is the energy derivative of the scattering time $\tau(\varepsilon)=1 / \gamma(\omega)$. The positive sign of $S$ in phase I indicates that the metallic properties are dominated by holelike carriers, since $\varepsilon^{\prime \prime}(k)<0$.

\section{B. Results for phase A-II}

Close to the transition temperature $T_{1}$ where Ni-OP, Co-OP, and Zn-OP transform into the phase A-II, the thermopower becomes negative and the temperature 
dependencies increase dramatically in magnitude. In agreement with the conductivity, $S$ gives evidence of semiconducting behavior, now dominated by electronlike transport. In an intrinsic semiconductor, conductivity and thermopower have contributions from both electrons $(e)$ and holes $(h):^{24}$

$$
\begin{aligned}
\sigma & =\sigma_{e}+\sigma_{h} \\
& =\sigma_{0} e-\frac{\Delta}{k_{B} T}
\end{aligned}
$$

and

$$
\begin{aligned}
S & =-\frac{\sigma_{e} S_{e}+\sigma_{n} S_{h}}{\sigma_{e}+\sigma_{h}} \\
& \left.=-\frac{k_{B}}{e} \frac{\sigma_{e}-\sigma_{h}}{\sigma_{e}+\sigma_{h}} \mid \frac{\Delta}{k_{B} T}+\Gamma\right) .
\end{aligned}
$$

In (5), the constant $\Gamma$ is a weighted sum of the kinetic terms, representing that the electrons and holes are distributed beyond the band edges. If this term is assumed to be temperature independent, the slopes of $S$ versus $1 / T$ will be

$$
\Delta_{S}=\frac{\sigma_{e}-\sigma_{h}}{\sigma_{e}+\sigma_{h}} \Delta
$$

where $\Delta$ is half the gap in the electronic spectrum. Values for $\Delta_{S}$ and $\Delta$ are difficult to derive accurately in the narrow temperature range between $T_{1}$ and $T_{2}$. In Ni-OP, the slopes of $\ln b$ and $S$ versus $1 / T$ give $\Delta \approx 250 \mathrm{meV}$ and $\Delta_{S} \approx 120 \mathrm{meV}$, respectively.

\section{Results for phase A-III}

In the vicinity of the $T_{2}$ transition there is a change in the slope of $S$ versus $1 / T$ as shown in Fig. 9, most notably observed in Ni-OP. However, the apparent activation energies $\Delta_{S}$ are surprisingly high for class- $A$ compounds in phase A-III compared to a simple chargedensity-wave state. In this case, the mobilities of electrons and holes may be calculated explicitly and since they are very similar, at least for simple band structures, $\Delta_{S}$ should be much lower than $\Delta$. As shown in Table $\mathrm{V}$, this is not the case and therefore the transport mechanisms at low temperatures in $M$-OP's are not as simple as assumed in the theories leading to (4)-(6). It is worth noting that $S$ versus $1 / T$ is not strictly linear even at the lowest temperatures. This indicates that the chemical potential is temperature dependent even below $T \simeq 150 \mathrm{~K}$, perhaps because of impurity levels in the gap.

\section{Results for phase B-II}

The thermopowers of Mg-OP and Mn-OP exhibit rather peculiar temperature dependences. Below 302 and 210 $\mathrm{K}$, respectively, $S$ becomes negative and eventually approaches an almost linear dependence on $1 / T$ with rather low activation energies $\Delta_{S}($ Table $\mathrm{V})$. However, at intermediate temperatures, the thermopower varies nonmonotonically, giving rise to local minima in $S$ at 284 and 185 $\mathrm{K}$ and local maxima at 265 and $132 \mathrm{~K}$ for the two compounds, respectively.

In order to determine whether these features of the thermopower in class- $B$ materials are associated with a sequence of phase transitions, we show in Fig. 10 the variations of both conductivity and thermopower of $\mathrm{Mg}-\mathrm{OP}$ and Mn-OP. In Mg-OP, the minima in both $\sigma$ and $S$ coincide with the structural transition temperature $T_{1}$. The features of both $\sigma$ and $S$ are therefore naturally ascribed to a strong temperature dependence of electron and hole mobilities, and perhaps also to the apparent gap in the electronic spectrum. In Mn-OP, our structural investigations indicate $T_{1}>300 \mathrm{~K}$, whereas $\sigma$ and $S$ indicate a transition at significantly lower temperatures. As mentioned above, Mn-OP crystals become damaged under $\mathrm{x}$ ray radiation which makes unambiguous conclusions difficult and we can offer no explanation for the behavior of Mn-OP. However, from the striking similarity between $\mathrm{Mg}-\mathrm{OP}$ and Mn-OP, we conclude that our classification is correct.

\section{E. Hysteresis effects}

In our presentation of the behavior of the conducting bis(oxalato)platinate with divalent metal cations, we have

\begin{tabular}{|c|c|c|c|c|c|c|}
\hline & \multicolumn{2}{|c|}{$\begin{array}{c}\text { Phase I } \\
\left(T>T_{1}\right)\end{array}$} & \multicolumn{2}{|c|}{$\begin{array}{c}\text { Phase II } \\
\left(T_{1}>T>T_{2}\right)\end{array}$} & \multicolumn{2}{|c|}{$\begin{array}{c}\text { Phase III } \\
\left(T_{2}>T\right)\end{array}$} \\
\hline & $\begin{array}{c}\sigma \\
\left(\Omega^{-1} \mathrm{~cm}^{-1}\right) \\
\end{array}$ & $\begin{array}{c}S \\
(\mu \mathrm{V} / \mathrm{K}) \\
\end{array}$ & $\begin{array}{c}\Delta \\
(\mathrm{meV}) \\
\end{array}$ & $\begin{array}{c}\Delta_{S} \\
(\mathrm{meV})\end{array}$ & $\begin{array}{c}\Delta \\
(\mathrm{meV}) \\
\end{array}$ & $\begin{array}{c}\Delta_{S} \\
(\mathrm{meV}) \\
\end{array}$ \\
\hline $\mathrm{Ni}-\mathrm{OP}$ & $\geq 100$ & & 240 & 120 & 60 & 70 \\
\hline Co-OP & $2-25$ & 6 & & & 50 & 40 \\
\hline $\mathrm{Zn}-\mathrm{OP}$ & $30-90$ & 7 & & & 55 & 25 \\
\hline $\mathrm{Mg}-\mathrm{OP}$ & $0.2-50$ & & 60 & 8 & & \\
\hline $\mathrm{Mn}-\mathrm{OP}$ & $10-47$ & 7 & 55 & 12 & & \\
\hline $\mathrm{KCP}(\mathrm{Br})$ & 300 & -1.5 & & & 72 & 5 \\
\hline
\end{tabular}
singled out three well-defined phases with characteristic

TABLE V. Summary of conduction and thermopower results from $M$-OP's compared to KCP. Phase I is metallic, phase II is a semiconductor due to cation ordering, and phase III is a chargedensity-wave semiconductor. 


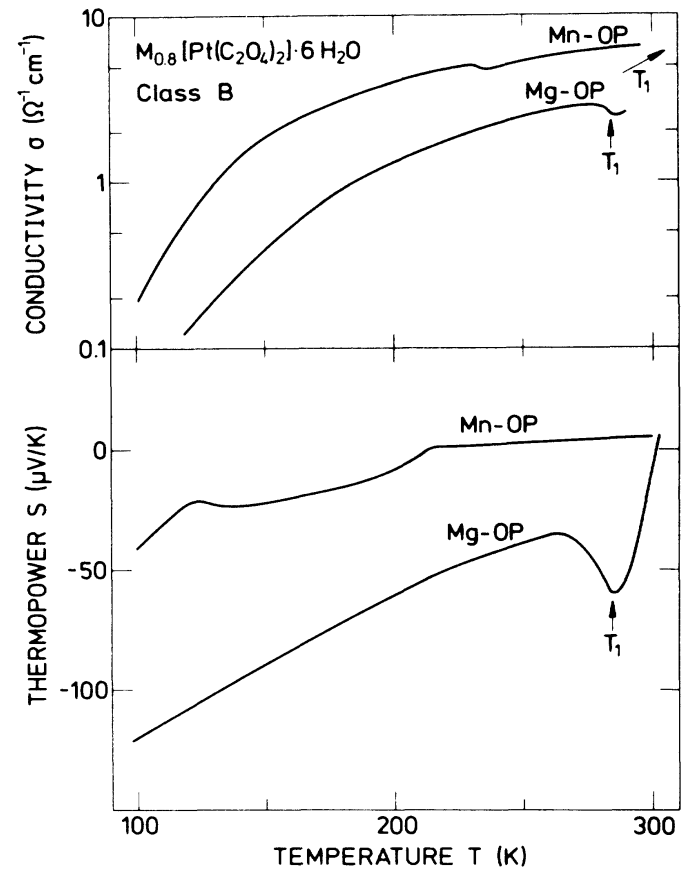

FIG. 10. Detailed view of the common features of class- $B$ $M$-OP's $(M=\mathrm{Mg}, \mathrm{Mn})$ with respect to their conduction and thermopower behavior. The molecular ordering transition temperature $T_{1}$ is indicated.

crystallographic and transport properties. Of course, it has not escaped our notice that these compounds have a long-standing reputation of producing multiphased crystals with properties that have been difficult to analyze. ${ }^{15,26}$ We are also aware of the fact that irreproducibility of results has often been ascribed to a dehydration problem occurring quite generally in platinum chain conductors. However, in the present study we have carefully monitored crystal quality and ensured ourselves of reproducibility of experimental results. Also, we have found that certain characteristic irregularities are independent of which cations are in use, despite the fact that the different $M$-OP's (e.g., $M=\mathrm{Zn}$ and $\mathrm{Mg}$ ) have very different tendencies to lose water. In consequence, we have come to the conclusion that these irregularities are caused by the competition between the tendency for the cations to order according to their mutual interaction and the tendency for them to arrange themselves according to the crystallographic lattice of $\left[\mathrm{Pt}\left(\mathrm{C}_{2} \mathrm{O}_{4}\right)_{2}\right]$ ions. The electronic transport properties are then understandable in terms of the coupling of the one-dimensional electron gas to the combined potential of the $\mathrm{Pt}$ lattice and the ordered $\left[\mathrm{M}\left(\mathrm{H}_{2} \mathrm{O}\right)_{6}\right]^{+2}$ cations. $^{27}$

The irregular behavior shows up as follows. In conductivity, hysteresis effects are pronounced in $\mathrm{Zn}-\mathrm{OP}$ and $\mathrm{Mg}-\mathrm{OP}$, in particular around the cation-ordering temperature $T_{1}$ (Table I), and evidence of chaotic behavior has been reported. ${ }^{19,26}$ Recently, a step-wise time dependence of $\sigma$ in $\mathrm{Mg}$-OP has been found, reminiscent of a devil'sstaircase behavior. ${ }^{27}$ Hysteresis in thermopower measurements are shown in Fig. 9. In Ni-OP, a hysteresis loop occurs around $T_{2}$, whereas in Mn-OP the hightemperature value of $S$ depends on the history of the sample. X-ray exposure times are in general quite long, so we have not been able to monitor the structural evolution in the "chaotic" temperature regions, but in Ni-OP both $T_{1}$ and $T_{2}$ have been difficult to determine. Finally, the coexistence of several superstructures in the B-II phase of $\mathrm{Mg}-\mathrm{OP}$ is a good fingerprint of competing interactions.

\section{DISCUSSION}

Based on the experimental results given above we will now turn to a comprehensive discussion of the behavior of the conducting bis(oxalato)platinates of divalent cations. We follow the same classification as used throughout this paper, namely that $M$-OP's have a common metallic phase with a one-dimensional Peierls distortion (phase I), but differ in their low-temperature behavior according to two different classes. Class- $\boldsymbol{A}$ compounds consisting of $\mathrm{Ni}-\mathrm{OP}, \mathrm{Co}-\mathrm{OP}$, and $\mathrm{Zn}-\mathrm{OP}$ first experience cation ordering below $T_{1}$ (phase A-II) which acts as a natural precursor for the Peierls transition at $T_{2}$ into the lowtemperature charge-density wave (phase A-III). On the other hand, in the $B$ class consisting of Fe-OP, Mg-OP, and Mn-OP, the symmetry of the ordered phase B-II is such that a Peierls transition is not accomplished. The four phases are discussed below.

\section{A. Phase I: Metallic band structure and transport}

By analogy to conducting tetracyanoplatinates, in particular $\mathrm{KCP}(\mathrm{Br})$, we expect the partially filled conduction band in $M$-OP's to originate from $d_{z^{2}}$ overlap between neighboring $\mathrm{Pt}$ ions. ${ }^{28}$ In $\mathrm{KCP}(\mathrm{Br})$, the band structure $\varepsilon(k)$ is indistinguishable from that of free electrons, i.e., $\varepsilon(k)=\hbar^{2} k^{2} /(2 m)$, shown in Fig. 11 in the simplified onedimensional Brillouin zone, corresponding to one $\mathrm{Pt}$ ion per unit cell. ${ }^{2,28}$

Two physical properties bear directly on the band structure at the Fermi level: optical properties and thermoelectric power. The former may, according to the Drude theory of metals, be parametrized into the plasma frequency $\omega_{p}$ which is related to the $\varepsilon(k)$ in the following way: ${ }^{20}$

$$
\begin{aligned}
\omega_{p}^{2} & =\frac{e^{2}}{\epsilon_{0} \hbar^{2}} \sum_{k}\left[f(\varepsilon(k)) \varepsilon^{\prime \prime}(k)\right]_{k=k_{F}} \\
& =\frac{2 e^{2} v_{F} n_{\mathrm{Pt}}}{\pi \epsilon_{0} \hbar} .
\end{aligned}
$$

In (7), $f(\varepsilon(k))$ is the Fermi-Dirac occupation number and $\varepsilon^{\prime \prime}(k)$ must be derived along the field polarization direction, i.e., the chain direction. $n_{\mathrm{Pt}}$ is the number of $\mathrm{Pt}$ chains per unit area perpendicular to c, and $v_{F}$ $\left(=\left[\hbar^{-1} \varepsilon^{\prime}(k)\right]_{k=k_{F}}\right)$ is the Fermi velocity. From the data of Table IV, a typical value of $\omega_{p} /(2 \pi c)$ for the $M$-OP's is $196000 \mathrm{~cm}^{-1}$, which gives $v_{F}=8.9 \times 10^{5} \mathrm{~m} / \mathrm{sec}$ or $\left[\varepsilon^{\prime}(k)\right]_{k=k_{F}}=5.9 \mathrm{eV \AA}$. These values are about $20 \%$ lower than for free electrons as measured in $\mathrm{KCP}(\mathrm{Br})$.

When we analyze the thermopower data at high temperatures according to (3), we find that it corresponds to 


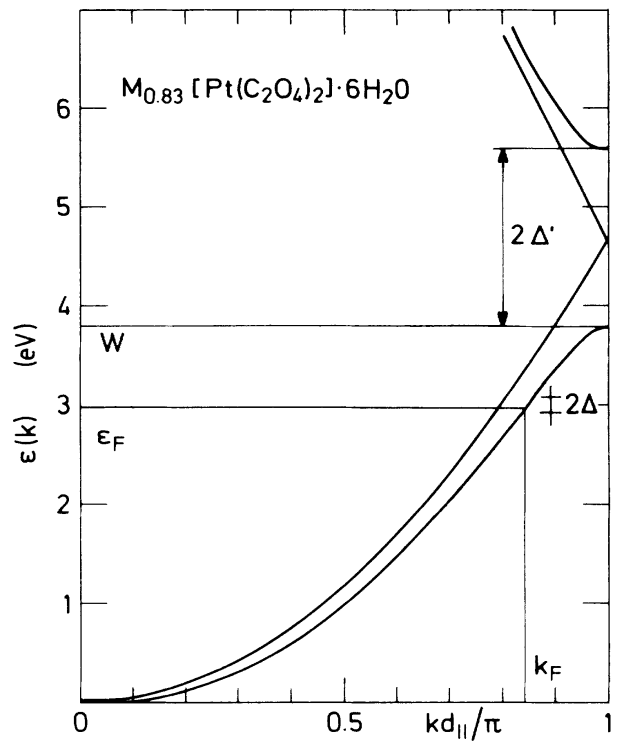

FIG. 11. Electronic band structures of $M$-OP. The zone boundary gap $2 \Delta^{\prime}$ is deduced from the measured properties as described in the text, and the gap $2 \Delta$ from the Peierls instability is also shown. The free-electron dispersion relation appropriate for $\mathrm{KCP}(\mathrm{Br})$ is shown for comparison.

an effective carrier mass $m^{*}=-(0.2-1) m$, where $m$ is the free-electron mass. Based on the measured values of $k_{F}, v_{F}\left(\left[=\hbar^{-1} \varepsilon^{\prime}(k)\right]_{k=k_{F}}\right)$, and $m^{*}\left(\left[=\hbar^{2} / \varepsilon^{\prime \prime}(k)\right]_{k=k_{F}}\right)$ it it possible to construct a plausible band structure $\varepsilon(k)$ for the $M$-OP's as follows. We assume that the deviation from free-electron behavior is caused by a gap $\Delta^{\prime}$ at the zone boundary $k=\pi / d_{\|}$and that the modified energydispersion relation is of the form:

$\varepsilon(k)=\frac{\hbar^{2}}{2 m}\left[\left[\frac{\pi}{d_{\|}}\right]^{2}-\left\{\left[k^{2}-\left(\pi / d_{\|}\right)^{2}\right]^{2}+\left(2 m \Delta^{\prime} / \hbar^{2}\right)^{2}\right\}^{1 / 2}\right]$.

Within this model, the measurements suggest that $\Delta^{\prime}=0.5 \pm 0.1 \mathrm{eV}$, giving an $\varepsilon(k)$ in phase I of the $M$-OP's as shown in Fig. 11. Hence, the metallic band structure of this class of compounds, as deduced from experimental results, is rather close to that based on theoretical calculations for KCP. ${ }^{28,29}$

\section{B. Phases A-II and B-II. Cation ordering and competing interactions}

In order to understand the differences in behavior between the class- $A$ and $-B$ compounds with respect to the cation ordering, we consider a simple model of one cation channel and one platinum chain. Left alone, the cations would order into a Wigner lattice of period $q_{M}$ where in the absence of $\mathrm{H}_{3} \mathrm{O}^{+}$one would have $q_{M}=k_{F}$. However, in the presence of the periodic potential of the crystallographic lattice with period $2 \pi / d$, the situation is more complicated. If the potential is sufficiently weak, the cations will form a Wigner lattice incommensurate with the Pt lattice, whereas a stronger potential will produce a ground-state cation configuration which will be commensurate with the lattice, but incommensurate with $k_{F}$. In order to satisfy the overall stoichiometry in the latter case, ordered domains are broken by misfit dislocations. The situation corresponds closely to the Frenkel-Kontorowa model and extensive theoretical studies ${ }^{30}$ have shown that this will lead to irregular behavior. We believe that our observations are in accord with these theoretical results.

Turning now to the electronic properties, the situation is markedly different for the two classes. In Ni-OP, Co$\mathrm{OP}$, and $\mathrm{Zn}-\mathrm{OP}$ (class $A$ ), the cations are ordered in each channel with a wave vector $k_{F}$ along c. However, as a result of the two symmetrically positioned channels around each chain, the electrons will sense a $2 k_{F}$ periodic potential along the chain. This opens a gap in the electronic spectrum at the Fermi level, giving rise to the semiconducting character of both conductivity $\sigma$ and thermopower $S$. (Note that this gap is caused by the cation ordering and not, as would be the case in a Peierls transition, by a distortion of the Pt-ion lattice.) The change in the sign of $S$ at $T_{1}$ indicates a more complicated change in $\varepsilon(k)$ than the occurrence of a simple gap.

By contrast, in $\mathrm{Mg}-\mathrm{OP}$ (class $B$ ) the conductivity $\sigma$ increases upon ordering at $T_{1}$, and Mn-OP shows similar behavior. This is because the cation ordering is incommensurate with $2 k_{F}$ and hence opens a gap away from the Fermi level, preserving metallic behavior. The ordering of the cations, however, increases the scattering time $\tau$ and hence $\sigma$.

The class- $B$ materials do not undergo a Peierls transition. This seems related to the fact that the B-II phase has a symmetry which is incompatible with a threedimensional charge-density wave. Nevertheless, their conductivity at low temperatures gives evidence of a gap at

TABLE VI. Relation between the crystallographic platinum lattice, the $\left[M\left(\mathrm{H}_{2} \mathrm{O}\right)_{6}\right]^{+2}$ cation lattice, and the $2 k_{F}$ electrons in the intermediate phase II of class $A$ (Ni-OP, Co-OP, and Zn-OP) and class $B$ (Fe-OP, Mg-OP, and Mn-OP). C is the commensurate; $\mathrm{I}$ is the incommensurate salts.

\begin{tabular}{lccc}
\hline B-II & Pt lattice & Cation lattice & $\begin{array}{c}2 k_{F} \\
\text { electrons }\end{array}$ \\
Pt-lattice & & $I$ & $I$ \\
cation-lattice & $C$ & $I$ & $C$ \\
$2 k_{F}$-electrons & $I$ & $I$ & \\
\hline \hline
\end{tabular}


the Fermi level which is indistinguishable from that observed in phase A-III. This occurs in the absence of any noticeable structural transition, but is followed by a complicated behavior in the thermopower. At present, we can offer no explanation of this low-temperature behavior in the B-II phase.

The relationships between the periods of the crystallographic $\mathrm{Pt}$ lattice, the $\left[M\left(\mathrm{H}_{2} \mathrm{O}\right)_{6}\right]^{+2}$ sublattice, and the $2 k_{F}$ electrons are summarized in Table VI.

\section{Phase A-III: Enhancement of the charge-density wave}

Below the transition $T_{1}$, class- $A$ compounds have properties which resemble closely those of a simple chargedensity-wave state. The electrons have a gap $2 \Delta$ at the Fermi level, and a concomitant distortion of wave vector $\mathbf{q}_{p}$ occurs in the Pt-ion lattice. It is therefore natural to return to Fig. 1 and address the question why $\Delta$ in the conducting bis(oxalato)platinates is significantly larger than in the tetracyanoplatinates. According to simple theory, the gap parameter increases with increasing electronic density of states in the metallic phase, and indeed the deduced $\varepsilon(k)$ shown in Fig. 10 suggest a density of states enhancement in $M$-OP's relative to KCP-type materials. Secondly, the external potential of the cations in $M$-OP's will increase appreciably the amplitude of the charge-density wave and hence $\Delta$.

\section{SUMMARY}

In summary, we have studied six linear platinum chain conductors of the type $M_{0.8}\left[\mathrm{Pt}\left(\mathrm{C}_{2} \mathrm{O}_{4}\right)_{2}\right] \cdot 6 \mathrm{H}_{2} \mathrm{O}$, where $M$ is in a divalent cation, by means of x-ray diffraction, dc conductivity, optical reflectivity, and thermopower measurements. When $M=\mathrm{Ni}, \mathrm{Co}, \mathrm{Zn}, \mathrm{Fe}, \mathrm{Mg}$, or $\mathrm{Mn}$, the $M$-OP salts form a common orthorhombic metallic phase at high temperatures. In this, "phase I," the Peierls instability gives rise to a one-dimensional distortion of the lattice in a similar way to that previously studied in the partially oxidized tetracyanoplatinates (TCP's). However, the free-electron behavior observed in the TCP's is somewhat modified in the $M$-OP's.

On cooling phase I, instead of showing the threedimensional ordering transition according to the Peierls instability, $M$-OP's exhibit a transition whose primary cause is the ordering of the $\left[M\left(\mathrm{H}_{2} \mathrm{O}\right)_{6}\right]^{+2}$ cations. This ordering may occur in two different ways. Class- $A M$ OP's ( $M=\mathrm{Ni}, \mathrm{Co}, \mathrm{Zn}$ ) form a phase A-II which is still of space group $C \mathrm{Ccm}$ and where the cation superlattice is characterized by wave vectors $\mathbf{q}_{M}=m\left(\mathbf{k}_{F}+\mathbf{a}^{*}\right)$ or $\mathbf{q}_{M}=m\left(\mathbf{k}_{F}+\mathbf{b}^{*}\right)$, where $m= \pm 1, \pm 2$. Class- $B$ com- ponents $(M=\mathrm{Fe}, \mathrm{Mg}, \mathrm{Mn})$ form a twinned monoclinic phase B-II, probably of space group $C 2 / c$ in which several cation superlattices may coexist. The different class- $B$ cation superlattices are all incommensurate with $a$ and $b$ and also most often with $k_{F}$.

The different symmetries of the superlattices of the phases A-II and B-II have determining effects on the transport behavior. Whenever the superstructure formation in A-II creates a gap in the electronic spectrum at the Fermi level and hence leads to semiconducting behavior or, in phase B-II metallic behavior prevails, at least just below the transition temperature $T_{1}$.

A third phase is formed in the class- $A$ compounds below a temperature $T_{2}$. This phase $A$-III is orthorhombic of space group Pccn, and interestingly the symmetry change with respect to phase A-II corresponds to the expected symmetry of the three-dimensional Peierls transition. We therefore associate phase A-III with the chargedensity-wave ground state common to one-dimensional conductors, but with an enhanced semiconducting gap due to the presence of the ordered cation lattice. A similar Peierls transition does not occur in the $B$ class, and we relate this observation to the fact that the cation superlattice is incommensurable with that corresponding to a threedimensional charge-density wave. Instead, these salts seem to gradually approach a semiconducting state on cooling without any change of crystal symmetry.

As a result of the competing tendencies towards ordering of the cations, either according to their mutual interactions or to their interaction with the Pt-ion lattice, the transitions between phases are not well behaved. In particular, class- $B$ compounds display both irregular hysteresis effects as well as the coexistence of several phases which may naturally be associated with such competing interactions in accord with theoretical results derived for this Frenkel-Kontorowa model.

\section{ACKNOWLEDGMENTS}

This work has been supported in part by the Danish Natural Science Research Council (A.B., K.C., K.M., C.S.J.), by the United Kingdom Sciences and Engineering Research Council (A.E.U., D.J.T., A.B.), and by a collaborative grant from North Atlantic Treaty Organization (NATO) (No. 016.81) (A.E.U., K.C.). It has also greatly profited from the hospitality extended by our laboratories to visiting collaborators. Useful contributions from, and discussions with D. M. Watkins and L. K. Hansen are gratefully acknowledged. C.S.J. was supported in part by the Royal Danish Academy of Sciences and Letters.
${ }^{*}$ Present address: Danish Institute of Fundamental Metrology, Building 322, Lundtoftevej 100, DK-2800 Lyngby, Denmark.

1J. M. Williams, A. J. Schultz, A. E. Underhill, and K. Carneiro, in Extended Linear Chain Compounds, edited by J. S. Miller (Plenum, New York, 1982), p. 73.

${ }^{2}$ A. E. Underhill, D. M. Watkins, J. M. Williams, and K. Carneiro, in Extended Linear Chain Compounds, edited by J. S.
Miller (Plenum, New York, 1982), p. 119.

${ }^{3}$ A. E. Underhill, D. J. Wood, and K. Carneiro, Synth. Methods 1, $395(1979 / 80)$.

${ }^{4}$ K. Krogmann, Z. Anorg. Allg. Chem. 358, 97 (1968).

${ }^{5}$ D. M. Watkins, A. E. Underhill, and C. S. Jacobsen, J. Phys. Chem. Solids 43, 183 (1982).

${ }^{6}$ A. J. Schultz, A. E. Underhill, and J. M. Williams, Inorg. 
Chem. 17, 1313 (1978).

${ }^{7}$ A. E. Underhill, D. M. Watkins, and C. S. Jacobsen, Solid State Commun. 36, 477 (1980).

${ }^{8}$ A. Bertinotti, D. Luzet, and A. Braude (unpublished).

${ }^{9}$ A. E. Underhill and D. J. Wood, in Quasi One-Dimensional Conductors II, 1978, Vol. 96 of Lecture Notes in Physics, edited by S. Barisic, A. Bjelis, J. R. Cooper, and B. Leontic (Springer-Verlag, Berlin, 1979), p. 208.

${ }^{10}$ A. Kobayashi, H. Kiondo, Y. Sasaki, H. Kobayashi, A. E. Underhill, and D. M. Watkins, Bull. Chem. Soc. Jpn. 55, 2074 (1982).

${ }^{11}$ A. Braude, A. Lindegaard-Anderson, and A. E. Underhill, Synth. Methods 10, 223 (1985).

${ }^{12}$ A. Braude, A. Lindegaard-Anderson, K. Carneiro, and A. E. Underhill, Synth. Methods 1, 35 (1979).

${ }^{13}$ A. Bertinotti, C. Carcaillet, G. Ichanno, and R. Saint-James, J. Phys. (Paris) 39, L307 (1978).

${ }^{14}$ A. Bertinotti and D. Luzet, J. Phys. (Paris) Colloq. 44, C31551 (1983).

${ }^{15}$ A. Braude, A. Lindegaard-Anderson, K. Carneiro, and A. S. Pedersen, Solid State Commun. 33, 365 (1980).

16J. Y. Dubois, Ph. D. thesis, University of Paris-Orsay, 1975.

${ }^{17}$ A. E. Underhill and D. J. Wood, Molecular Metals, NATO $A S I$, edited by W. E. Hatfield (Plenum, New York, 1978), p. 377.
${ }^{18}$ H. J. Pedersen and A. E. Underhill, Solid State Commun. 33 289 (1980).

${ }^{19}$ M. Mizuno, A. E. Underhill, and K. Carneiro, J. Phys. C 16, 2105 (1983).

${ }^{20} \mathrm{~F}$. Wooten, Optical Properties of Solids (Academic, New York, 1972), p. 197.

${ }^{21}$ D. Kuse and H. R. Zeller, Phys. Rev. Lett. 27, 1060 (1971).

${ }^{22}$ L. H. Greene, D. B. Tanner, A. J. Epstein, and J. S. Miller, Phys. Rev. B 25, 1331 (1982).

${ }^{23}$ J. J. Hopfield, Phys. Rev. 137, A419 (1965).

${ }^{24}$ P. M. Chaikin, J. F. Kwak, T. W. Jones, A. F. Garrito, and A. J. Heeger, Phys. Rev. Lett. 31, 601 (1973).

${ }^{25}$ See, e.g., J. M. Ziman, Electrons and Phonons (Oxford University Press, Oxford, 1962).

${ }^{26} \mathrm{~K}$. Carneiro and A. E. Underhill, J. Phys. (Paris) Colloq. 44, C3-1007 (1983).

${ }^{27}$ K. Carneiro, L. K. Hansen, A. Brande, K. Mortensen, C. S. Jacobsen, D. Turner, and A. E. Underhill, Mol. Cryst. Liq. Cryst. 120, 413 (1985).

${ }^{28}$ H. Yersin and G. Gliemann, Ann. Acad. Sci. (N.Y.) 313, 539 (1978).

${ }^{29}$ R. P. Messmer and D. R. Salahub, Phys. Rev. Lett. 35, 533 (1975).

${ }^{30}$ P. Bak, Rep. Prog. Phys. 45, 587 (1982). 


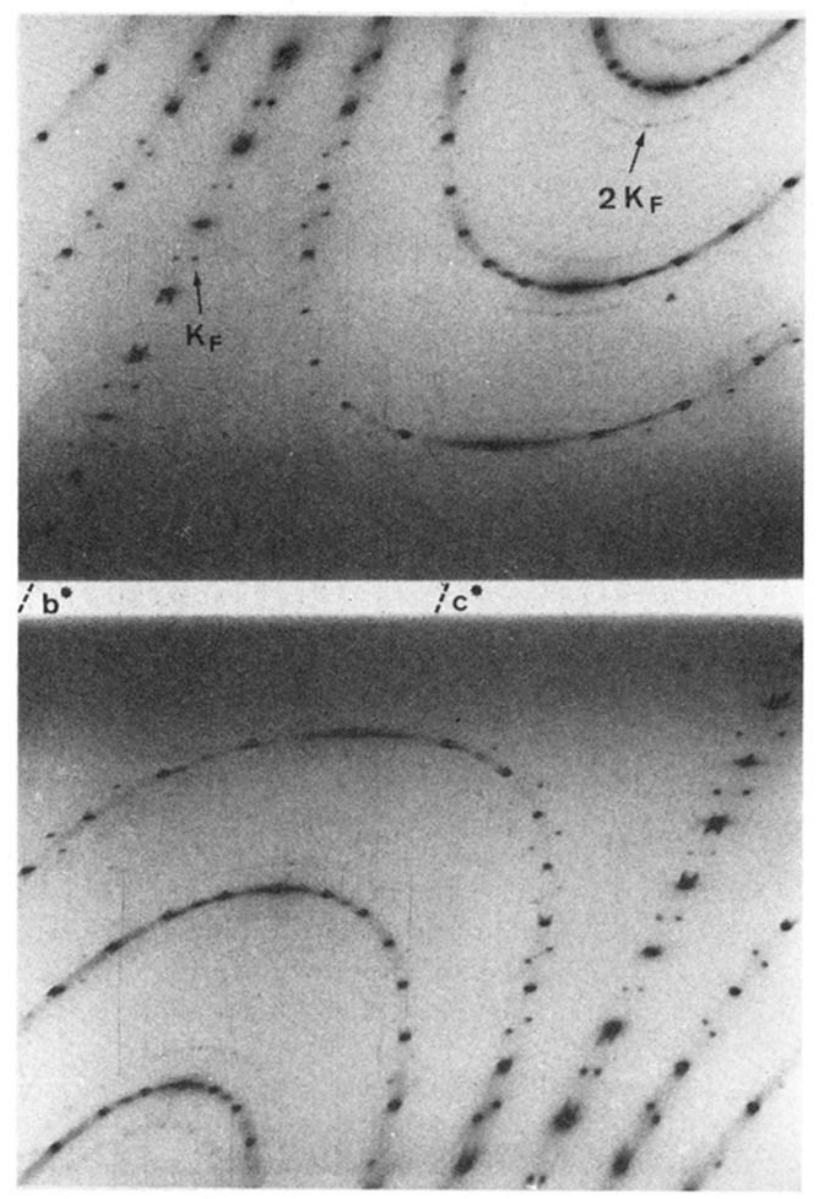

FIG. 6. X-ray Weissenberg recording of $\mathrm{Zn}-\mathrm{OP}$ at $283 \mathrm{~K}$. It shows the $(0 \mathrm{KL})$ plane, containing $2 \mathbf{k}_{F}$ diffuse scattering and second-order satellites, both surrounding strong Bragg-reflection layer lines, as well as first-order spots surrounding extinct Bragg reflections. The picture is characteristic of phase A-II, as shown in Fig. 4. 


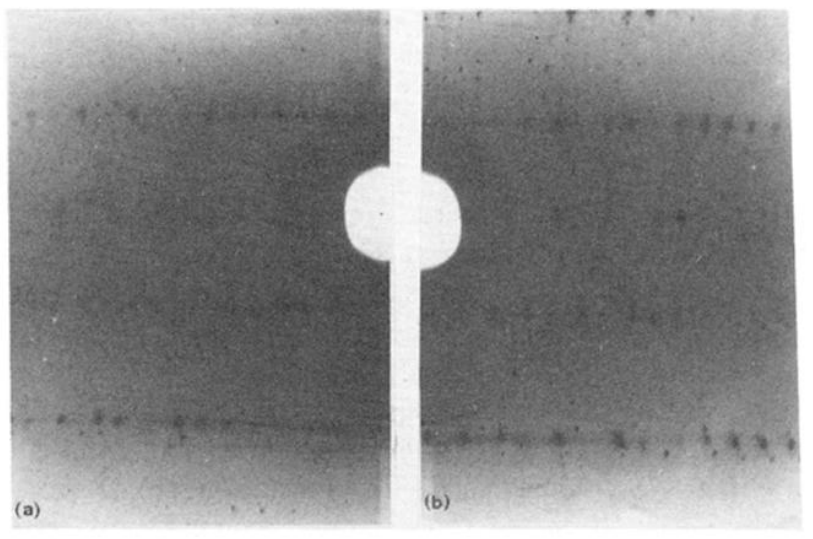

FIG. 7. X-ray photographs showing two different superstructures in one crystal of $\mathrm{Mg}-\mathrm{OP}$ at $230 \mathrm{~K}$. The two pictures were obtained by focusing the $\mathrm{x}$-ray beam on different parts of the crystal. 